\title{
The Value of Preoperative Systemic Inflammatory Response index (SIRI) Combined with Albumin- Bilirubin Grading (ALBI) in Predicting Early Recurrence Following Curative Resection of Single Hepatocellular Carcinoma
}

\section{Yubin Fu}

Zhengzhou University First Affiliated Hospital

\section{Zhengkai Feng}

Zhengzhou University First Affiliated Hospital

Jiahao Xue

Zhengzhou University First Affiliated Hospital

\section{Penglei Ge}

Zhengzhou University First Affiliated Hospital

\section{Lin Li}

Zhengzhou University First Affiliated Hospital

Yang Wu ( $\sim$ sunny2000@yeah.net)

Zhengzhou University First Affiliated Hospital

\section{Research Article}

Keywords: systemic inflammatory response index, albumin-bilirubin grading, SIRI, ALBI, early recurrence, hepatocellular carcinoma, HCC, curative resection

Posted Date: October 25th, 2021

DOI: https://doi.org/10.21203/rs.3.rs-970759/v1

License: (c) (i) This work is licensed under a Creative Commons Attribution 4.0 International License.

Read Full License 


\section{Abstract}

Background: This study was conducted to explore the risk factors for early recurrence of single hepatocellular carcinoma (HCC) following curative resection, and to evaluate the predictive value of systemic inflammatory response index (SIRI) combined with the albumin - bilirubin index (ALBI) for early recurrence of HCC following curative resection.

Methods: We retrospectively analyzed the clinical data of 233 patients with pathologically confirmed HCC who were admitted to the First Affiliated Hospital of Zhengzhou University from January 2015 to January 2018. The optimal cut-off values of alpha-fetoprotein, neutrophil-to-lymphocyte ratio (NLR), monocyte-tolymphocyte ratio (MLR), platelet-to-lymphocyte ratio (PLR), SIRI, and ALBI were determined by receiver operating characteristic curve (ROC), and the predictive efficiency of each index was compared by area under curve (AUC). A Cox-proportional hazard regression model was used to analyze factors affecting early recurrence. Kaplan-Meier analysis was used to estimate the 2-year recurrence-free survival (RFS) rate of different factors.

Results: The AUC of SIRI for predicting early recurrence was 0.644 , which was better than that of NLR, MLR, PLR, and SII. SIRI > 0.96, ALBI $>-2.7$, tumor diameter $>5 \mathrm{~cm}$, and microvascular invasion (MVI) were risk factors for early recurrence after radical resection of HCC. The AUC of SIRI combined with ALBI was 0.759 , which was better than that of single SIRI and ALBI. Combined with the above four risk factors, a new prognostic index was constructed, and the AUC for predicting early recurrence was 0.801 .

Conclusion: SIRI, ALBI, MVI, and tumor diameter could be considered reliable predictors of early recurrence following curative resection of single HCC. Preoperative SIRI combined with ALBI possesses an important reference value in predicting early recurrence following radical resection of HCC. It is helpful for clinicians in identifying high-risk patients with early recurrence, actively carry out postoperative adjuvant treatment, and improving the prognosis of patients.

\section{Background}

Primary liver cancer is the sixth most common malignant tumor and the fourth leading cause of tumor death worldwide [1]; it is the fourth most common malignant tumor and the second leading cause of tumor in China, of which hepatocellular carcinoma (HCC) accounts for 85-90\% [2]. For patients with single HCC in Barcelona clinic liver cancer (BCLC) stage 0 or stage $A$, radical surgical resection is an important means of achieving long-term survival [3]. However, the recurrence and metastasis rate of patients with $\mathrm{HCC}$ is as high as $40-70 \% 5$ years following radical resection, which is the leading cause of death [4-6]. Among them, early recurrence (recurrence within 2 years following surgery) accounts for more than $70 \%$ of recurrent tumors, and the prognosis is even worse [7]. Therefore, it is particularly important to evaluate the risks of early recurrence following radical resection of $\mathrm{HCC}$, and to screen the population at high risk of early recurrence. 
Tumor-related inflammation and immune responses are essential for the occurrence, development, angiogenesis, and metastasis of malignant tumors [8]. Several studies have shown that preoperative scores based on routine blood inflammatory indicators, such as neutrophil-to-lymphocyte ratio (NLR), monocyte-to-lymphocyte ratio (MLR), and platelet-to-lymphocyte ratio (PLR), affect the prognosis of HCC $[5,9,10]$. In 2016, Qi proposed a new inflammatory index based on peripheral blood lymphocytes, neutrophils, and monocytes, namely the systemic inflammatory response index (SIRI), which has been proven to predict the prognosis of many types of malignant tumors, such as pancreatic cancer, cervical cancer, esophageal gastric cancer, and breast cancer [11-14]. However, the value of SIRI in predicting early recurrence following radical resection of HCC has not been reported.

Liver function critically affects the treatment and prognosis of patients with HCC [15], it is particularly important to completely evaluate liver function. The Child-Pugh class has been widely applied in the evaluation of liver function and some tumor staging systems; however, the ability to evaluate liver function in patients with HCC with good liver reserve is limited, and is subjectively influenced by ascites and hepatic encephalopathy $[16,17]$. Albumin - bilirubin index (ALBI) grade is a newly proposed alternative method for evaluating liver function based on albumin and bilirubin, which can avoid the influence of subjective factors and can further divide Child-Pugh A patients with HCC into two subgroups with different prognoses $[18,19]$. Some studies have confirmed that the ALBI grade is better than the Child-Pugh grade in predicting the prognosis of patients with $\operatorname{HCC}[16,17,20]$ and proposed to combine it with conventional prognostic models [21,22]. However, there are few studies on the value of ALBI in predicting early recurrence following radical resection of HCC.

This study aimed to explore the risk factors of early recurrence of HCC following radical resection, clarify the value of SIRI combined with ALBI in predicting early recurrence following radical resection of HCC, and construct a new prognostic index $(\mathrm{PI})$ to predict early recurrence and evaluate its effect.

\section{Methods}

\section{Inclusion and Exclusion Criteria}

\section{Inclusion}

1. The ages of the patients were $\geq 18$ years,

2. Child-Pugh grade A or B returned to grade A following hepatoprotective treatment before surgery,

3. HCC was diagnosed for the first time, and no antineoplastic therapy was administered before the operation,

4. Single tumors (BCLC stage 0 or stage $A$ ),

5. Radical resection of HCC,

6. No intervention, radiofrequency, radiotherapy, or systematic treatment was performed within 2 months following the operation. 
1. Patients with incomplete clinical and pathological data,

2. Presence of hematological diseases or other malignant tumors,

3. Accompanied by acute or subacute infectious diseases,

4. Rupture of the liver cancer

\section{General features}

This study retrospectively analyzed the data of 233 patients with HCC who underwent radical resection at the First Affiliated Hospital of Zhengzhou University from January 2015 to January 2018.

We collected the demographic data of the patients, including sex, age, and history of hypertension and diabetes. The laboratory data within one week before surgery, including hepatitis B surface antigen (HBsAg), alanine aminotransferase (ALT), aspartate aminotransferase (AST), total bilirubin (Tbil), albumin (Alb), alpha-fetoprotein (AFP), peripheral blood leukocyte (WBC), neutrophils, monocytes, lymphocytes, and platelets, were obtained. In terms of tumor characteristics, tumor diameter, liver cirrhosis, microvascular invasion (MVI), and Edmondson-Steiner grade of HCC were considered as possible influencing factors following resection.

The calculation formula for each score was as follows: NLR= neutrophil count $(\times 109) /$ lymphocyte count $(\times 109), M L R=$ monocyte count $(\times 109) /$ lymphocyte count $(\times 109)$, PLR= platelet count $(\times 109) /$ lymphocyte count $(\times 109)$, SIRI $=$ monocyte count $(\times 109) \times$ neutrophil count $(\times 109 / \mathrm{L}) /$ lymphocyte count $(\times 109 / \mathrm{L}) /$ lymphocyte count $(\times 109 / \mathrm{L}), \mathrm{ALBI}=-0.085 \times \mathrm{Alb}+0.66 \times \mathrm{Log} 10 \mathrm{Tbil}(\mu \mathrm{mol} / \mathrm{L})$.

\section{Treatment and follow-up}

All 233 patients with HCC underwent curative resection. A total of 217 patients underwent laparotomy. Among them, 180 underwent non-anatomical resection and 37 underwent anatomical resection. The remaining 16 patients underwent laparoscopic hepatectomy.

\section{Criteria for curative resection}

(1) no macroscopic tumor thrombi were noted in the hepatic vein, portal vein, bile duct, and inferior vena cava

(2) In the absence of adjacent organ involvement, portal lymph nodes, or distal metastases

(3) The surgical margin and tumor boundary are $>1 \mathrm{~cm}$, or histologic examination of the cross section of the resected liver is free of residual tumor cells, which is a negative surgical margin [2]

\section{Criteria of recurrence}

New intrahepatic or extrahepatic lesions were detected according to imaging examinations, including ultrasound, enhanced computed tomography (CT), magnetic resonance imaging(MRI), contrast-enhanced 
ultrasound, and positron emission tomography-CT. Early recurrence was defined as recurrence within 2 years following radical resection of HCC.

\section{Follow-up}

Postoperative follow-up was performed via telephone and outpatient review. The start time of follow-up was the start time of surgery, and the end point was the 2-year recurrence-free survival (RFS), which was defined as the date from the beginning of surgery to the date of tumor recurrence within 2 years. The last follow-up was performed on May 2021.

\section{Statistical analysis}

All the analyses were performed using the IBM SPSS Statistics software (version 26). A receiver operating characteristic (ROC) curve was constructed to determine the optimal cut-off value. Continuous variables are presented as mean \pm standard deviation, and categorical variables as frequencies (\%). A Coxproportional hazard regression model was used to analyze the factors affecting the early recurrence of HCC. ROC curve analysis was used to evaluate the performance in predicting the early recurrence of HCC. Survival curves were plotted using the Kaplan-Meier method and compared using the log-rank test. Statistical significance was set at $p<0.05$.

\section{Results}

\section{Clinical features}

A total of 233 patients were included in this study according to the inclusion and exclusion criteria. There were 184 men $(79 \%)$ and 49 women (21\%), with an average age of $53.7 \pm 10.3 \mathrm{y}$. Before the operation, 193 patients (82.8\%) had complicated hepatitis B, and 202 patients (91.8\%) had cirrhosis. All the patients had solitary tumors with an average diameter of $5.0 \pm 3.0 \mathrm{~cm}$. All the patients had Child-Pugh grade A preoperatively. A total of 113 patients (48.5\%) had early recurrence, 105 patients (92.9\%) had intrahepatic recurrence, and 8 patients had extrahepatic recurrence, including 4 cases of lung metastasis, 2 cases of kidney metastasis, 1 case of omental metastasis, and 1 case of lymph node metastasis (Table 1 ). 
Table 1

General data of 233 patients with single hepatocellular carcinoma

\begin{tabular}{|l|}
\hline \\
\end{tabular}




\begin{tabular}{|c|c|}
\hline & n (\%) \\
\hline male & 184(79\%) \\
\hline female & $49(21 \%)$ \\
\hline Age(years) & $53.7 \pm 10.3$ \\
\hline$<60$ & $162(69.5 \%)$ \\
\hline$\geq 60$ & $71(30.5)$ \\
\hline \multicolumn{2}{|l|}{ Hypertension } \\
\hline no & $187(80.3 \%)$ \\
\hline yes & $46(19.7 \%)$ \\
\hline \multicolumn{2}{|l|}{ Diabetes } \\
\hline no & $214(91.8 \%)$ \\
\hline yes & $19(8.2 \%)$ \\
\hline \multicolumn{2}{|l|}{ Liver cirrhosis } \\
\hline yes & $202(86.7 \%)$ \\
\hline no & $31(13.3 \%)$ \\
\hline \multicolumn{2}{|l|}{ Surgical methods } \\
\hline laparotomy & $217(93.1 \%)$ \\
\hline laparoscopic & $16(6.9 \%)$ \\
\hline nepatectomy & $5.0 \pm 3.0$ \\
\hline Tumor diameter(cm) & $136(58.4 \%)$ \\
\hline $\begin{array}{l}\leq 5 \\
>5\end{array}$ & $97(41.6 \%)$ \\
\hline $\begin{array}{l}\text { Edmondson-Steiner } \\
\text { grade }\end{array}$ & $180(77.3 \%)$ \\
\hline$\nabla-\nabla$ & $53(22.7 \%)$ \\
\hline$\nabla-\nabla$ & \\
\hline Microvascular invasion & $182(78.1 \%)$ \\
\hline yes & $51(21.9 \%)$ \\
\hline no & \\
\hline
\end{tabular}




\begin{tabular}{|ll|}
\hline & $\mathbf{n}(\%)$ \\
\hline Non-early recurrence & $120(51.5 \%)$ \\
Early recurrence & $113(48.5 \%)$ \\
Intrahepatic recurrence & $105(45.1 \%)$ \\
Lung metastasis & $4(1.7 \%)$ \\
Kidney metastasis & $2(0.9 \%)$ \\
Omental metastasis & $1(0.4 \%)$ \\
Lymph node & $1(0.4 \%)$ \\
metastasis & \\
\hline
\end{tabular}

The optimal cut-off value and area under the curve (AUC) of AFP, ALBI and inflammatory indicators before surgery

The ROC curve analysis demonstrated that $A F P=35, A L B I=-2.7, S I R I=0.96, P L R=75.36, N L R=2.47$ and $M L R=0.3$ were the optimal cut-off values. According to the threshold value of each indicator, patients with values less than or equal to the cut-off value were divided into the low-value group, while patients with values larger than the cut-off value were divided into the high-value group. The characteristics of the laboratory examination data after grouping are presented in Table 2. The prognostic value of these inflammatory indicators was further analyzed by comparing the AUC. The results demonstrated that the AUC of SIRI was larger than that of NLR, PLR, and MLR, indicating that the prognostic value of SIRI was better than that of NLR, PLR, and MLR (Table 3). 
Table 2

The characteristics of the laboratory examination data after grouping of 233 patients with HCC.

\begin{tabular}{|c|c|}
\hline 口ם & frequencies $(\%) /$ mean \pm standard deviation \\
\hline HBsAg & $193(82.8 \%)$ \\
\hline+ & $40(17.2 \%)$ \\
\hline \multicolumn{2}{|l|}{-} \\
\hline ALT & $156(67 \%)$ \\
\hline$\leq 40$ & 77(33\%) \\
\hline \multicolumn{2}{|l|}{$>40$} \\
\hline AST & $161(69.1 \%)$ \\
\hline$\leq 40$ & $72(30.9 \%)$ \\
\hline \multicolumn{2}{|l|}{$>40$} \\
\hline AFP & $121(51.9 \%)$ \\
\hline$<35$ & $112(48.1 \%)$ \\
\hline \multicolumn{2}{|l|}{$\geq 35$} \\
\hline SIRI & $128(54.9 \%)$ \\
\hline$\leq 0.96$ & $105(45.1 \%)$ \\
\hline \multicolumn{2}{|l|}{$>0.96$} \\
\hline ALBI & $113(48.5 \%)$ \\
\hline$\leq-2.7$ & $120(51.5 \%)$ \\
\hline \multicolumn{2}{|l|}{$>-2.7$} \\
\hline PLR & $97(41.6 \%)$ \\
\hline$\leq 75.36$ & $136(58.4 \%)$ \\
\hline \multicolumn{2}{|l|}{$>75.36$} \\
\hline NLR & $168(72.1 \%)$ \\
\hline$\leq 2.47$ & $65(27.9 \%)$ \\
\hline \multicolumn{2}{|l|}{$>2.47$} \\
\hline MLR & $127(54.5 \%)$ \\
\hline$\leq 0.3$ & $106(45.5 \%)$ \\
\hline$>0.3$ & $40.1 \pm 4.1$ \\
\hline
\end{tabular}




\begin{tabular}{|ll|}
\hline Albumin $(\mathrm{g} / \mathrm{L})$ & frequencies $(\%) /$ mean \pm standard deviation \\
Total bilirubin $(\mu \mathrm{mol} / \mathrm{L})$ & $12.9 \pm 6.6$ \\
White blood cell count $\left(\times 10^{9}\right)$ & $134.4 \pm 62.5$ \\
Platelet count $\left(\times 10^{9}\right)$ & $3.1 \pm 1.3$ \\
Neutrophil count $\left(\times 10^{9}\right)$ & $1.5 \pm 0.6$ \\
Lymphocyte count $\left(\times 10^{9}\right)$ & $0.5 \pm 0.2$ \\
Monocyte count $\left(\times 10^{9}\right)$ & \\
\hline
\end{tabular}

Table 3

The optimal cut-off value and area under the curve (AUC) of AFP, ALBI and inflammatory indicators before surgery.

\begin{tabular}{|llllll|}
\hline variables & Cut-off value & sensitivity(\%) & specificity(\%) & Youden index & AUC (95\% Cl) \\
\hline AFP & 35 & 54.9 & 58.3 & 0.132 & $0.576(0.502-0.649)$ \\
\hline ALBI & -2.7 & 72.6 & 67.5 & 0.401 & $0.654(0.582-0.726)$ \\
\hline SIRI & 0.96 & 63.7 & 72.5 & 0.362 & $0.644(0.572-0.716)$ \\
PLR & 75.36 & 63.7 & 46.7 & 0.104 & $0.528(0.453-0.602)$ \\
NLR & 2.47 & 40.7 & 84.2 & 0.249 & $0.637(0.565-0.708)$ \\
MLR & 0.3 & 53.1 & 63.3 & 0.164 & $0.587(0.514-0.660)$ \\
\hline
\end{tabular}

Univariate and multivariate analysis of early recurrence following radical resection of HCC.

Cox univariate analysis showed that preoperative AFP, NLR, MLR, SIRI, ALBI, tumor diameter, EdmondsonSteiner grade, and microvascular invasion had significant effects on the early recurrence of HCC. The significant factors in the Cox univariate analysis were included in the Cox multivariate regression model. The results showed that SIRI, ALBI, tumor diameter, and microvascular invasion were independent influencing factors of early recurrence of HCC (Table 4). 
Table 4

Univariate and multivariate analysis of early recurrence following radical resection of HCC.

\begin{tabular}{|c|c|c|c|c|}
\hline \multirow[t]{2}{*}{ Variables } & \multicolumn{2}{|l|}{ Univariate analysis } & \multicolumn{2}{|c|}{ Multivariate analysis } \\
\hline & $\mathrm{HR}(95 \% \mathrm{Cl})$ & $\mathbf{P}$ & $\mathrm{HR}(95 \% \mathrm{Cl})$ & $\mathbf{P}$ \\
\hline Gender & $1.210(0.781-1.875)$ & 0.394 & & \\
\hline Age & $1.278(0.867-1.884)$ & 0.215 & \multirow{2}{*}{$\begin{array}{l}2.167(1.293- \\
3.633)\end{array}$} & 0.003 \\
\hline HBsAg & $0.947(0.584-1.536)$ & 0.827 & & 0.001 \\
\hline ALT & $1.082(0.734-1.596)$ & 0.689 & $\begin{array}{l}2.441(1.460- \\
4.081)\end{array}$ & 0.038 \\
\hline AST & $1.362(0.928-1.999)$ & 0.114 & \multirow{3}{*}{$\begin{array}{l}1.497(1.023- \\
2.190) \\
2.163(1.420- \\
3.296)\end{array}$} & \multirow[t]{12}{*}{$<0.001$} \\
\hline AFP & $1.509(1.042-2.186)$ & 0.030 & & \\
\hline SIRI & $2.936(1.996-4.318)$ & $<0.001$ & & \\
\hline ALBI & $3.161(2.095-4.768)$ & $<0.001$ & & \\
\hline PLR & $1.450(0.988-2.128)$ & 0.058 & & \\
\hline NLR & $2.444(1.674-3.568)$ & $<0.001$ & & \\
\hline MLR & $1.494(1.033-2.163)$ & 0.033 & & \\
\hline Surgical method & $0.812(0.378-1.746)$ & 0.594 & & \\
\hline Liver cirrhosis & $0.925(0.537-1.593) 1.520 \otimes 1.050-$ & 0.779 & & \\
\hline Tumor diameter & & 0.026 & & \\
\hline \multirow{2}{*}{$\begin{array}{l}\text { Edmondson-Steiner } \\
\text { grading }\end{array}$} & \multirow{2}{*}{$3.104(2.102-4.585)$} & 0.002 & & \\
\hline & & $<0.001$ & & \\
\hline
\end{tabular}

Value of SIRIALBI combination in predicting early recurrence

SIRI and ALBI were combined to divide patients into four groups: low SIRI+ low ALBI group, low SIRI+ high ALBI group, high SIRI+ low ALBI group, and high SIRI+ high ALBI group. ROC curves were constructed based on SIRI, ALBI, and SIRI-ALBI (Figure 1). By comparing their AUC, it was found that SIRI combined with ALBI was superior to SIRI and ALBI in predicting early recurrence of $\mathrm{HCC}(\mathrm{AUC}=0.759, p<0.001)$ (Figure 1).

Establishing a new PI for predicting early recurrence following curative resection of HCC

The tumor diameter, SIRI, ALBI, and MVI were included in the Cox-proportional hazard regression model to fit the early recurrence risk function, $h(t)=h_{0} \exp (0.896 \times S I R I+0.894 \times A L B I+0.39 \times$ tumor diameter $+0.817 \times \mathrm{MVI})$. Constructing the (PI of early recurrence following curative resection of HCC, 
$\mathrm{PI}=0.896 \times \mathrm{SIRI}+0.894 \times \mathrm{ALBI}+0.39 \times$ tumor diameter +0.817×MVI, SIRI > 0.96, ALBI > -2.7, tumor diameter > $5 \mathrm{~cm}$, and MVI were all assigned 1; otherwise, 0 was assigned. The ROC curve was constructed to determine the optimal PI cut-off value of 1.25, and patients were divided into two groups: low-risk group $(\mathrm{PI}<1.25$ and high-risk group $(\mathrm{PI} \geq 1.25)$. ROC analysis was used to evaluate the predictive value of the prognostic index. The results showed that the AUC of PI was 0.801 , indicating a high predictive value (Figure 1). Kaplan-Meier curve analysis showed that the 2-year RFS rate of the low-risk group was higher than that of the high-risk group $(p<0.001)$ (Figure 2).

\section{2-year RFSsurvival curve based on tumor diameter, SIRI, ALBI, and MVI}

The 2-year RFS survival curves of tumor diameter, MVI, SIRI, ALBI, and SIRI-ALBI were plotted using the Kaplan-Meier curves, and the differences in survival curves were tested using log-rank tests. The results demonstrated that patients with tumor diameter $>5 \mathrm{~cm}$, SIRI >0.96, ALBI > -2.7, and MVI had significantly lower recurrence-free survival at 2 years following surgery than those with tumor diameter $\leq 5 \mathrm{~cm}$ $(p=0.023)$, no microvascular invasion $(p<0.001)$, $\mathrm{SIRI} \leq 0.96(p<0.001)$, and ALBI $\leq-2.7(p<0.001)$ (Figure 3). Patients with high SIRI-high ALBI had significantly lower relapse-free survival at 2 years following surgery than patients with low SIRI-low ALBI $(p<0.001)$ (Figure 4).

\section{Discussion}

At present, there is no consensus on the cut-off point for the time period for early recurrence following radical resection of HCC. Some clinical practice guidelines and most studies consider 2 years as the time cut-off point to distinguish early recurrence from late recurrence [3, 7, 23-29]. This study demonstrated that most patients (67.7\%) showed recurrence within 2 years following surgery, and the risk of recurrence decreased and tended to lever off after 2 years. Therefore, the time of early recurrence was set within 2 years following surgery in this study. Studies have confirmed that early recurrence and late recurrence are independent entities caused by different risk factors [6]. Early recurrence is considered to be intrahepatic or multicentric metastasis of the primary tumor, and is related to the invasiveness of the primary tumor, such as tumor number, tumor size, MVI, tumor differentiation, microsatellite nodules, and AFP [5, 6, 30], while late recurrence is mainly based on the etiology and background of liver cirrhosis [26, 28]. The results of multivariate analysis demonstrated that tumor diameter $>5 \mathrm{~cm}$ and $\mathrm{MVI}$ were independent risk factors for early recurrence of HCC following radical resection; however, there was no significant difference in the degree of tumor differentiation.

As one of the most common indicators for the diagnosis and prognosis of HCC, AFP still has a controversial predictive value for early postoperative recurrence [26]. We found that AFP had a poor 
predictive effect on early recurrence of HCC, with an AUC of only 0.576 . Multivariate analysis also indicated that AFP could not be used as an independent factor influencing early recurrence of HCC following curative resection $(p>0.05)$. This result is consistent with previous studies $[5,6,30]$, whether AFP could be used as a predictor needs to be further verified.

In recent years, an increasing number of studies have demonstrated that inflammation plays an important role in the occurrence, development, and metastasis of malignant tumors $[13,25,31]$. The potential mechanisms could be that neutrophils are mainly concentrated in the peritumoral matrix of liver cancer tissues, [32] and can release angiogenic factors and inflammatory mediators, such as interleukin-1 $\beta$ (IL-1 $\beta$ ), interleukin-6 (IL-6), tumor necrosis factor-a (TNF-a), and reactive oxygen species (ROS). In addition, neutrophils can inhibit the cytolytic activity of immune cells, which promotes the proliferation and metastasis of cancer cells $[5,9,10,31,33]$. Neutrophils and tumor-associated macrophages modulate the suppression of anti-tumor immunity by inhibiting the immune function of natural killer cells and $\mathrm{T}$ cells, leading to malignant progression [5]. Lymphocytes play an anticancer role in host immunity by inducing apoptosis and inhibiting the migration and invasion of cancer cells [10]. Platelets could protect tumor cells from natural killer cell-mediated lysis, and induce rapid activation of neutrophils, thereby promoting metastasis $[28,31]$.

The majority of HCC cases occur in the context of hepatitis and cirrhosis; therefore, inflammation is particularly significant in the process of its occurrence and development. Preoperative scoring systems based on peripheral inflammatory indicators, such as NLR, MLR, and PLR, have also been proven to be associated with the prognosis of HCC $[5,9,10]$. In 2016, Qi proposed a new inflammatory index based on peripheral blood lymphocytes, neutrophils, and monocytes, namely SIRI [11], and it has been proven to predict the prognosis of various malignant tumors, such as pancreatic cancer, cervical cancer, esophageal cancer of the stomach, and breast cancer [12-14]; however, its prognostic value in HCC has not been studied. In this study, patients with HCC complicated with hematological diseases and infectious diseases were excluded to prevent them from influencing routine blood indicators and interfere with the predictive value of early recurrence. The results showed that SIRI, NLR, and MLR could all be used to predict the early recurrence of single HCC following radical surgery, except for PLR. However, only SIRI was an independent risk factor for early postoperative recurrence. ROC curve analysis showed that among these indices, SIRI was of higher value in predicting early recurrence of single HCC following radical resection.

Child-Pugh grade was proposed by Child, Turcotte, and Paugh and used to evaluate the liver function of patients with liver cirrhosis and portal hypertension [34,35]. Since then, it has been widely applied to evaluate the liver function reserve of patients with liver disease and has been applied in various tumor staging systems, including American Joint Committee on Cancer tumor/node/metastasis (AJCC TNM) staging, BCLC staging, and China liver cancer staging (CNLC) staging. The Child-Pugh score system is composed of five indicators: bilirubin, albumin, PT, ascites, and hepatic encephalopathy, in which ascites and hepatic encephalopathy are highly subjective, and the degree of ascites is correlated with albumin concentration, decreasing the ability to evaluate liver function reserve $[16,17]$. In addition, with the 
constant improvement of antiviral therapy and diagnosis of HCC, the number of patients with early HCC with good liver function has increased [15]. In this study, the preoperative Child-Pugh grades of 233 patients were all grade A; therefore, it cannot be applied to predict early recurrence of HCC.

ALBI, which was put forward in 2015, is a new index evaluating liver function in patients with HCC, especially those with good liver function reserve. $[16,18]$. This index is only composed of bilirubin and albumin, which are both convenient and objective markers. Many studies have proved that its prediction effect is better than that of the Child-Pugh grade. In this study, 233 patients were divided into two groups by $A L B I$, and the $A B L I>-2.7$ group indicated that the prognosis was worse, which further confirmed the previous study.

We speculate that the combination of SIRI and ALBI can improve the prediction of early recurrence following curative resection, because the combination can simultaneously evaluate the inflammation, immune status, and liver function of patients with HCC. In this study, SIRI combined with ALBI was superior to SIRI or ALBI alone in predicting early recurrence in patients with single HCC. Patients in the low SIRI-low ALBI group had a lower 2-year recurrence-free survival rate. Therefore, preoperative SIRI combined with $A L B I$ is an important reference value for predicting early recurrence of $\mathrm{HCC}$ following radical resection.

At present, there is no consensus on risk stratification tools for the early postoperative recurrence of HCC. Except for the AJCC-TNM tumor staging system in the United States, most tumor staging systems are not from surgically treated patients and do not consider MVI $[26,36]$. This study showed that the area under the ROC curve for TNM staging was only 0.6 , which could be due to the Child-Pugh scoring system used for liver function evaluation in the TNM system, while the Child-Pugh grading of 233 patients with HCC in this study was all grade A before surgery; thus, the difference in liver function between the two groups could not be effectively distinguished. In addition, the TNM-tumor staging system lacks evaluation of tumor-related inflammatory indicators. This study combined preoperative SIRI and ALBI to construct a new PI and confirmed that it is of higher value in predicting early postoperative recurrence.

There are certain limitations to this study. First, this study is a single-center retrospective study, which is prone to selection bias. Second, the cut-off value of each index was selected by the ROC curve. Different cut-off values could affect the final statistical results and warrants further verification. Therefore, further multicenter prospective studies should be conducted.

\section{Conclusions}

Therefore, SIRI, ALBI, MVI, and tumor diameter could be considered reliable predictors of early recurrence following curative resection of single HCC. Preoperative SIRI combined with ALBI possesses an important reference value in predicting early recurrence following radical resection of HCC. It is helpful for clinicians in identifying high-risk patients with early recurrence, actively carry out postoperative adjuvant treatment, and improving the prognosis of patients. 


\section{Abbreviations}

HCC

hepatocellular carcinoma

BCLC

Barcelona clinic liver cancer

NLR

neutrophil-to-lymphocyte ratio

MLR

monocyte-to-lymphocyte ratio

PLR

platelet-to-lymphocyte ratio

SIRI

systemic inflammatory response index

ALBI

Albumin - bilirubin index

PI

prognostic index

HBsAg

hepatitis B surface antigen

ALT

alanine aminotransferase

AST

aspartate aminotransferase

Tbil

total bilirubin

Alb

albumin

AFP

alpha-fetoprotein

WBC

peripheral blood leukocyte

MVI

microvascular invasion

RFS

recurrence-free survival time

ROC

receiver-operating curve

AUC

area under the curve 


\section{Declarations}

\section{Ethics approval and consent to participate}

This study was approved by the Ethics Committee of The First Affiliated Hospital of Zhengzhou University, No. 1 Jianshe East Road, Zhengzhou, Henan Province, China. All procedure performed in studies were in accordance with the Declaration of Helsinki.

\section{Consent for publication}

This study was approved by the Ethics Committee of The First Affiliated Hospital of Zhengzhou University, No. 1 Jianshe East Road, Zhengzhou, Henan Province, China.

Since the study was conducted anonymously, no consent to publication by the patient was required.

\section{Availability of data and materials}

The data that support the fundings of this study are available from the corresponding author (Yang Wu, $\mathrm{MD}, \mathrm{PhD}$ ) on request.

\section{Competing interests}

All our authors have no conflicts of interest to disclose.

\section{Funding}

This research was supported by Joint Construction Project of Henan Medical Science and Technology Research Program (grant number LHGJ20190136).

\section{Authors' contributions}

Yubin Fu and Penglei Ge put forward the idea of this study and collected, analyzed the data and wrote the article. Yubin Fu, Zhengkai Feng searched literatures. Penglei Ge, Lin Li and Yang Wu reviewed the manuscript and gave important professional advice. Yubin Fu, Zhengkai Feng, Jiahao Xue wrote the article. Yang Wu approved the final version of the article. Yubin Fu is the guarantor.

\section{Acknowledgements}

This research was supported by Joint Construction Project of Henan Medical Science and Technology Research Program (grant number LHGJ20190136).

This study was performed at the First Affiliated Hospital of Zhengzhou University, Zhengzhou. This study used data from the First Affiliated Hospital of Zhengzhou University, Zhengzhou.

We would like to thank Editage (www.editage.cn) for English language editing. 


\section{References}

1. Bray F, Ferlay J, Soerjomataram I, Siegel RL, Torre LA, Jemal A. Global cancer statistics 2018: GLOBOCAN estimates of incidence and mortality worldwide for 36 cancers in 185 countries. Cancer J Clin. 2018;68(6):394-424.

2. Zhou J, Sun H, Wang Z, Cong W, Wang J, Zeng M, Zhou W, Bie P, Liu L, Wen T, et al: Guidelines for the Diagnosis and Treatment of Hepatocellular Carcinoma (2019 Edition). Liver cancer 2020, 9(6):682720.

3. EASL Clinical. Practice Guidelines: Management of hepatocellular carcinoma. Journal of hepatology. 2018;69(1):182-236.

4. Zhou SL, Zhou ZJ, Hu ZQ, Song CL, Luo YJ, Luo CB, Xin HY, Yang XR, Shi YH, Wang Z, et al. Genomic sequencing identifies WNK2 as a driver in hepatocellular carcinoma and a risk factor for early recurrence. Journal of hepatology. 2019;71(6):1152-63.

5. Wu Y, Tu C, Shao C. Inflammatory indexes in preoperative blood routine to predict early recurrence of hepatocellular carcinoma after curative hepatectomy. BMC Surg. 2021;21(1):178.

6. Xu W, Li R, Liu F. Novel Prognostic Nomograms for Predicting Early and Late Recurrence of Hepatocellular Carcinoma After Curative Hepatectomy. Cancer management research. 2020;12:1693-712.

7. Cha DI, Jang KM, Kim SH, Kim YK, Kim H, Ahn SH. Preoperative Prediction for Early Recurrence Can Be as Accurate as Postoperative Assessment in Single Hepatocellular Carcinoma Patients. Korean J Radiol. 2020;21(4):402-12.

8. Greten FR, Grivennikov SI. Inflammation and Cancer: Triggers, Mechanisms, and Consequences. Immunity. 2019;51(1):27-41.

9. Chen Q, Li F, Zhong C, Zou Y, Li Z, Gao Y, Zou Q, Xia Y, Wang K, Shen F. Inflammation Score System using Preoperative Inflammatory Markers to Predict Prognosis for Hepatocellular Carcinoma after Hepatectomy: A Cohort Study. J Cancer. 2020;11(17):4947-56.

10. Zeng J, Zeng J, Wu Q, Lin K, Zeng J, Guo P, Zhou W, Liu J. Novel inflammation-based prognostic nomograms for individualized prediction in hepatocellular carcinoma after radical resection. Annals of translational medicine. 2020;8(17):1061.

11. Qi Q, Zhuang L, Shen Y, Geng Y, Yu S, Chen H, Liu L, Meng Z, Wang P, Chen Z. A novel systemic inflammation response index (SIRI) for predicting the survival of patients with pancreatic cancer after chemotherapy. Cancer. 2016;122(14):2158-67.

12. Chen Y, Jin M, Shao Y, Xu G. Prognostic Value of the Systemic Inflammation Response Index in Patients with Adenocarcinoma of the Oesophagogastric Junction: A Propensity Score-Matched Analysis. Disease markers. 2019;2019:4659048.

13. Chao B, Ju X, Zhang L, Xu X, Zhao Y. A Novel Prognostic Marker Systemic Inflammation Response Index (SIRI) for Operable Cervical Cancer Patients. Frontiers in oncology. 2020;10:766. 
14. Hua X, Long ZQ, Huang X, Deng JP, Wen W, He ZY, Guo L, Zhang WW, Lin HX. The preoperative systemic inflammation response index (SIRI) independently predicts survival in postmenopausal women with breast cancer. Curr Probl Cancer. 2020;44(4):100560.

15. Hiraoka A, Kumada T, Michitaka K, Kudo M. Newly Proposed ALBI Grade and ALBI-T Score as Tools for Assessment of Hepatic Function and Prognosis in Hepatocellular Carcinoma Patients. Liver cancer. 2019;8(5):312-25.

16. Kumada T, Toyoda H, Tada T, Yasuda S, Tanaka J. Changes in Background Liver Function in Patients with Hepatocellular Carcinoma over 30 Years: Comparison of Child-Pugh Classification and Albumin Bilirubin Grade. Liver cancer. 2020;9(5):518-28.

17. Pan J, Chen S, Tian G, Jiang T. Preoperative Albumin-Bilirubin Grade With Prognostic Nutritional Index Predicts the Outcome of Patients With Early-Stage Hepatocellular Carcinoma After Percutaneous Radiofrequency Ablation. Frontiers in medicine. 2020;7:584871.

18. Johnson PJ, Berhane S, Kagebayashi C, Satomura S, Teng M, Reeves HL, O'Beirne J, Fox R, Skowronska A, Palmer D, et al. Assessment of liver function in patients with hepatocellular carcinoma: a new evidence-based approach-the ALBI grade. Journal of clinical oncology: official journal of the American Society of Clinical Oncology. 2015;33(6):550-8.

19. Dong ZR, Zou J, Sun D, Shi GM, Ke AW, Cai JB, Sun HC, Qiu SJ, Li T, Zhou J, et al. Preoperative Albumin-Bilirubin Score for Postoperative Solitary Hepatocellular Carcinoma within the Milan Criteria and Child-Pugh A Cirrhosis. J Cancer. 2017;8(18):3862-7.

20. Peng Y, Wei Q, He Y, Xie Q, Liang Y, Zhang L, Xia Y, Li Y, Chen W, Zhao J, et al. ALBI versus child-pugh in predicting outcome of patients with HCC: A systematic review. Expert Rev Gastroenterol Hepatol. 2020;14(5):383-400.

21. Pinato DJ, Sharma R, Allara E, Yen C, Arizumi T, Kubota K, Bettinger D, Jang JW, Smirne C, Kim YW, et al. The ALBI grade provides objective hepatic reserve estimation across each BCLC stage of hepatocellular carcinoma. Journal of hepatology. 2017;66(2):338-46.

22. Ma XL, Zhou JY, Gao XH, Tian L, Wu J, Zhang CY, Zhou Y, Dai Q, Wang BL, Pan BS, et al. Application of the albumin-bilirubin grade for predicting prognosis after curative resection of patients with earlystage hepatocellular carcinoma. Clin Chim Acta. 2016;462:15-22.

23. Heimbach JK, Kulik LM, Finn RS, Sirlin CB, Abecassis MM, Roberts LR, Zhu AX, Murad MH, Marrero JA. AASLD guidelines for the treatment of hepatocellular carcinoma. Hepatology. 2018;67(1):35880.

24. Lee S, Kang TW, Song KD, Lee MW, Rhim H, Lim HK, Kim SY, Sinn DH, Kim JM, Kim K, et al. Effect of Microvascular Invasion Risk on Early Recurrence of Hepatocellular Carcinoma After Surgery and Radiofrequency Ablation. Annals of surgery. 2021;273(3):564-71.

25. Zhu Y, Gu L, Chen T, Zheng G, Ye C, Jia W. Factors influencing early recurrence of hepatocellular carcinoma after curative resection. J Int Med Res. 2020;48(8):300060520945552.

26. Chan AWH, Zhong J, Berhane S, Toyoda H, Cucchetti A, Shi K, Tada T, Chong CCN, Xiang BD, Li LQ, et al. Development of pre and post-operative models to predict early recurrence of hepatocellular 
carcinoma after surgical resection. Journal of hepatology. 2018;69(6):1284-93.

27. Kobayashi T, Aikata H, Kobayashi T, Ohdan H, Arihiro K, Chayama K. Patients with early recurrence of hepatocellular carcinoma have poor prognosis. Hepatobiliary Pancreat Dis Int. 2017;16(3):279-88.

28. Portolani N, Coniglio A, Ghidoni S, Giovanelli M, Benetti A, Tiberio GA, Giulini SM. Early and late recurrence after liver resection for hepatocellular carcinoma: prognostic and therapeutic implications. Annals of surgery. 2006;243(2):229-35.

29. Wen L, Weng S, Yan C, Ye R, Zhu Y, Zhou L, Gao L, Li Y. A Radiomics Nomogram for Preoperative Prediction of Early Recurrence of Small Hepatocellular Carcinoma After Surgical Resection or Radiofrequency Ablation. Frontiers in oncology. 2021;11:657039.

30. Kim WJ, Lim TW, Park PJ, Choi SB, Kim WB. Prognostic markers affecting the early recurrence of hepatocellular carcinoma with liver cirrhosis after curative resection. Int J Biol Mark. 2019;34(2):123-31.

31. Yu JI, Park HC, Yoo GS, Choi C, Choi MS, Nam H, Baek SY, Park M. Clinical importance of the absolute count of neutrophils, lymphocytes, monocytes, and platelets in newly diagnosed hepatocellular carcinoma. Scientific reports. 2021;11(1):2614.

32. Kuang DM, Zhao Q, Wu Y, Peng C, Wang J, Xu Z, Yin XY, Zheng L. Peritumoral neutrophils link inflammatory response to disease progression by fostering angiogenesis in hepatocellular carcinoma. Journal of hepatology. 2011;54(5):948-55.

33. Qin L, Li C, Xie F, Wang Z, Wen T. Are inflammation-based markers useful in patients with hepatocellular carcinoma and clinically significant portal hypertension after liver resection? Bioscience trends. 2020;14(4):297-303.

34. Child CG, Turcotte JG. Surgery and portal hypertension. Major problems in clinical surgery. 1964;1:185.

35. Pugh RN, Murray-Lyon IM, Dawson JL, Pietroni MC, Williams R. Transection of the oesophagus for bleeding oesophageal varices. Br J Surg. 1973;60(8):646-9.

36. Amin MB, Greene FL, Edge SB, Compton CC, Gershenwald JE, Brookland RK, Meyer L, Gress DM, Byrd DR, Winchester DP. The Eighth Edition AJCC Cancer Staging Manual: Continuing to build a bridge from a population-based to a more "personalized" approach to cancer staging. Cancer J Clin. 2017;67(2):93-9.

\section{Figures}




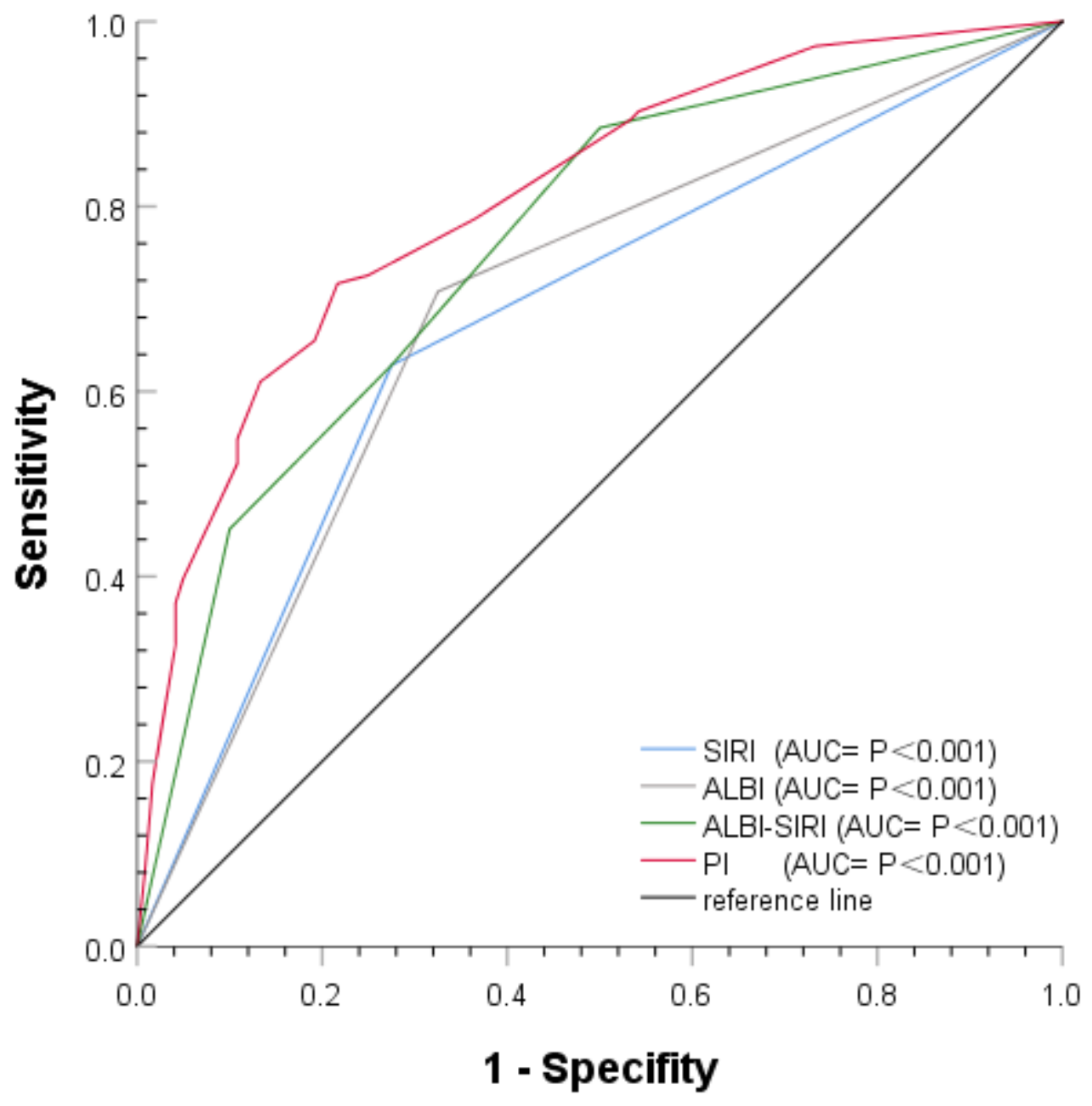

Figure 1

Comparison of systemic inflammatory response index (SIRI), albumin-bilirubin index (ALBI), ALBI-SIRI, and prognostic index $(\mathrm{PI})$ in predicting early recurrence. 


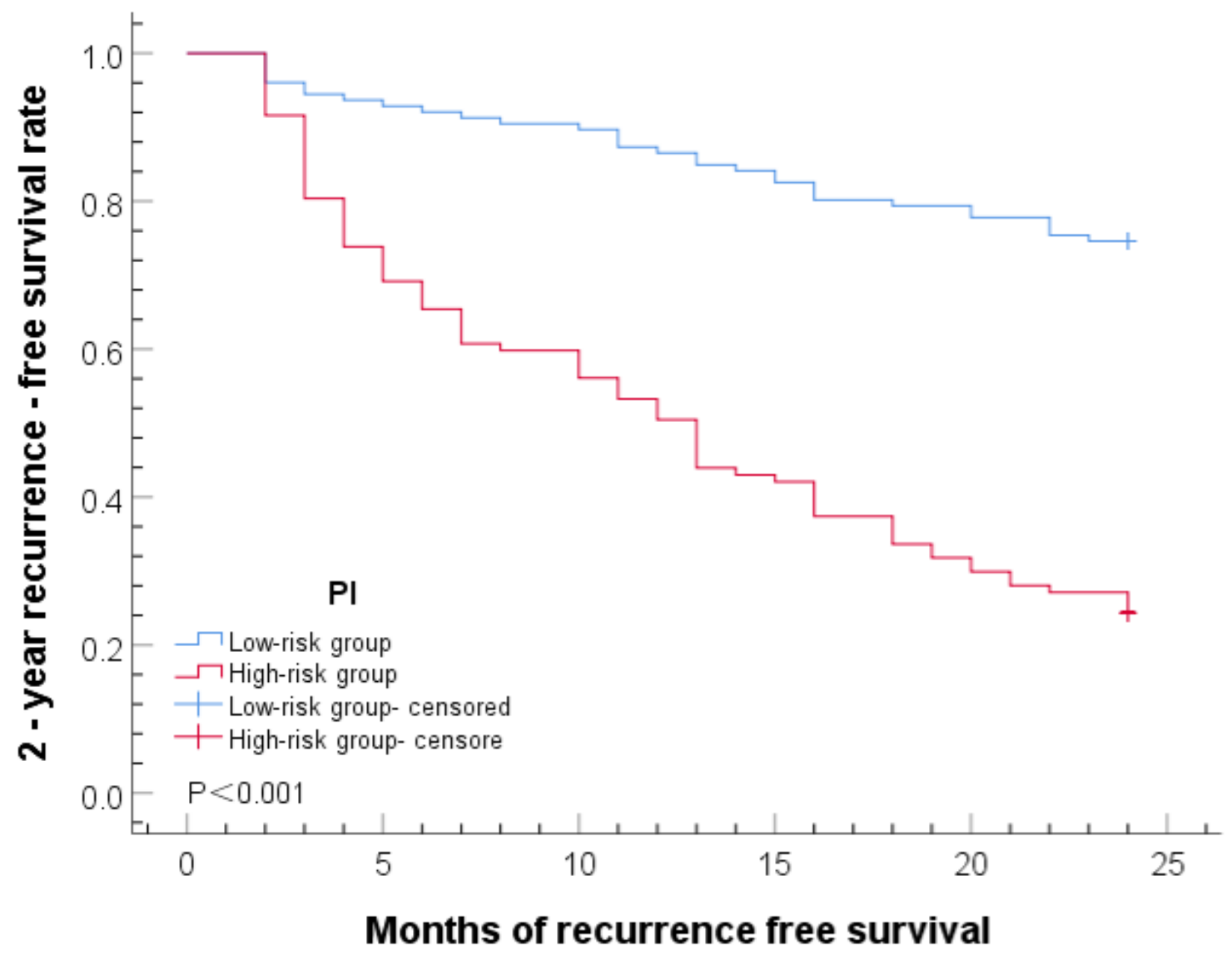

Figure 2

2-year recurrence-free survival curves of patients with low-risk group and high-risk group. 

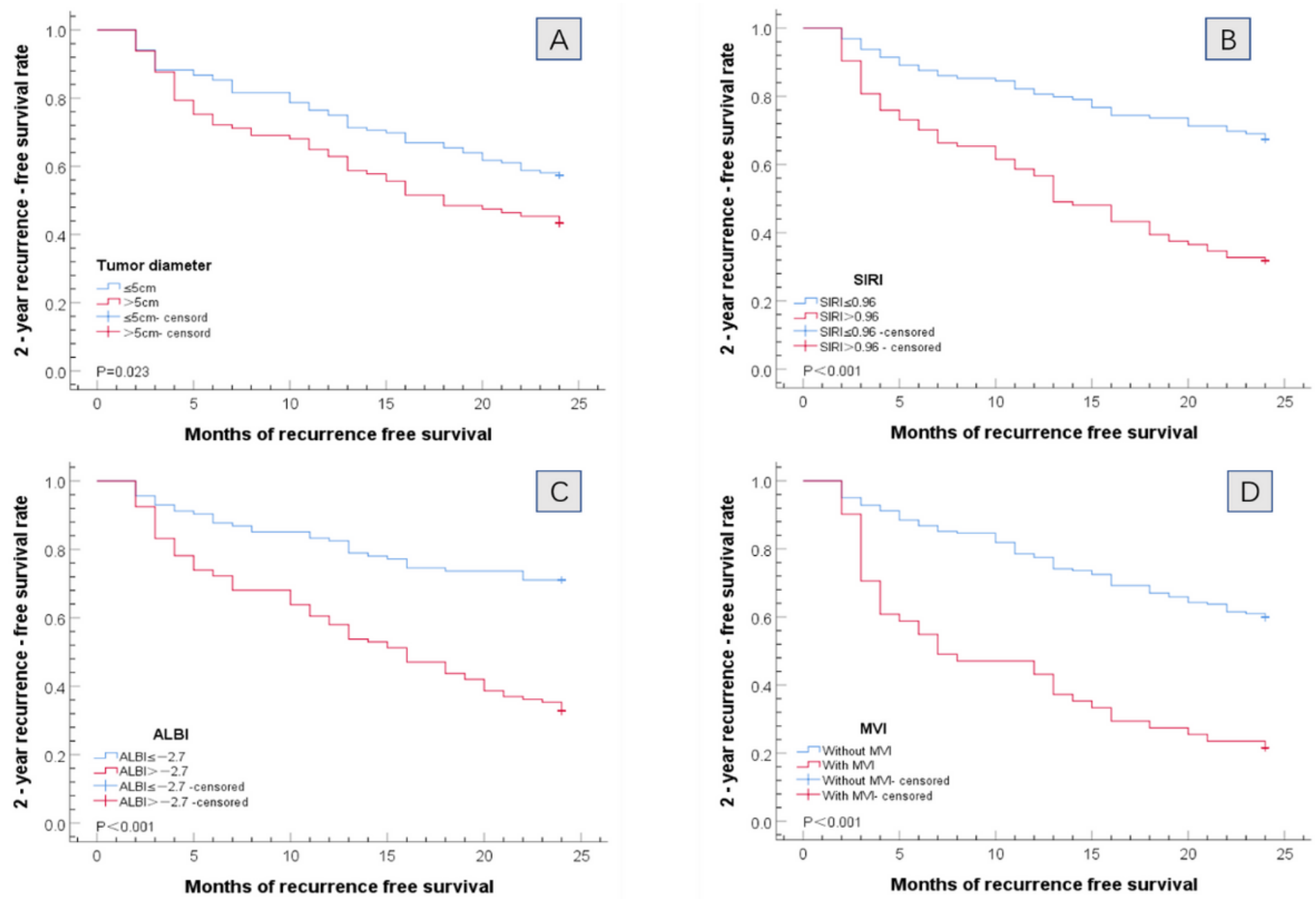

Figure 3

A) 2-year recurrence-free survival curves of patients with tumor diameter $\leq 5 \mathrm{~cm}$ and $₫ 5 \mathrm{~cm}$. B) 2-year recurrence-free survival curves of patients with systemic inflammatory response index (SIRI) $\leq 0.96$ and

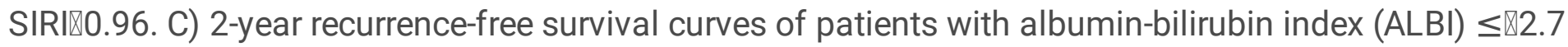
and ALBIه囚2.7. D) 2-year recurrence-free survival curves of patients with microvascular invasion (MVI). 


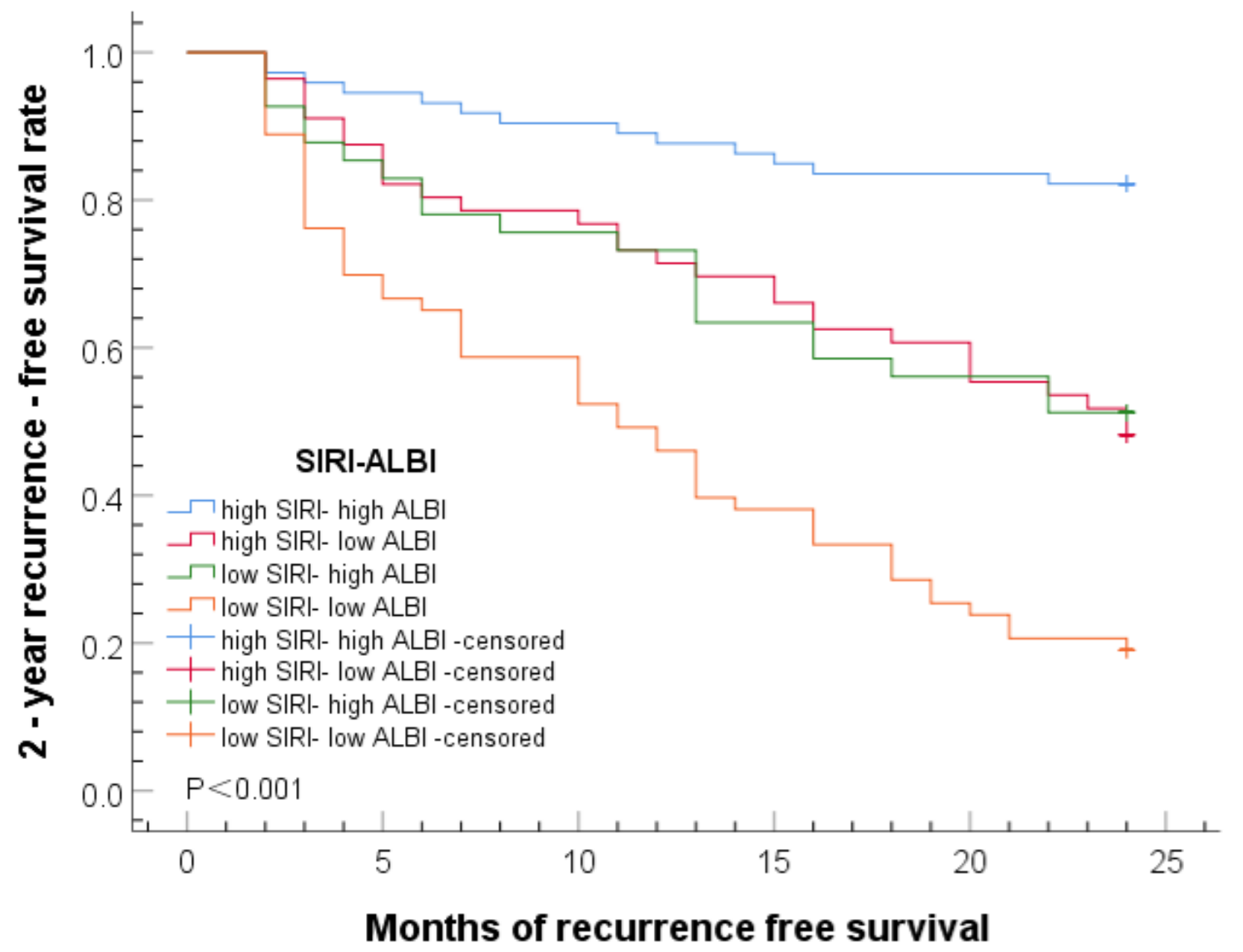

Figure 4

2-year recurrence-free survival curves of patients with systemic inflammatory response index (SIRI) combined with albumin-bilirubin index (ALBI). 